\title{
Pathological neural networks and artificial neural networks in ALS: diagnostic classification based on pathognomonic neuroimaging features
}

\author{
Peter Bede ${ }^{1,2} \mathbb{D} \cdot$ Aizuri Murad ${ }^{1} \cdot$ Orla Hardiman $^{1}$
}

Received: 20 August 2021 / Revised: 7 September 2021 / Accepted: 9 September 2021 / Published online: 28 September 2021

(c) The Author(s) 2021

\begin{abstract}
The description of group-level, genotype- and phenotype-associated imaging traits is academically important, but the practical demands of clinical neurology centre on the accurate classification of individual patients into clinically relevant diagnostic, prognostic and phenotypic categories. Similarly, pharmaceutical trials require the precision stratification of participants based on quantitative measures. A single-centre study was conducted with a uniform imaging protocol to test the accuracy of an artificial neural network classification scheme on a cohort of 378 participants composed of patients with ALS, healthy subjects and disease controls. A comprehensive panel of cerebral volumetric measures, cortical indices and white matter integrity values were systematically retrieved from each participant and fed into a multilayer perceptron model. Data were partitioned into training and testing and receiver-operating characteristic curves were generated for the three study-groups. Area under the curve values were 0.930 for patients with ALS, 0.958 for disease controls, and 0.931 for healthy controls relying on all input imaging variables. The ranking of variables by classification importance revealed that white matter metrics were far more relevant than grey matter indices to classify single subjects. The model was further tested in a subset of patients scanned within 6 weeks of their diagnosis and an AUC of 0.915 was achieved. Our study indicates that individual subjects may be accurately categorised into diagnostic groups in an observer-independent classification framework based on multiparametric, spatially registered radiology data. The development and validation of viable computational models to interpret single imaging datasets are urgently required for a variety of clinical and clinical trial applications.
\end{abstract}

Keywords Neuroradiology $\cdot$ Machine learning $\cdot$ Amyotrophic lateral sclerosis $\cdot$ Neuroimaging $\cdot$ Artificial neural networks

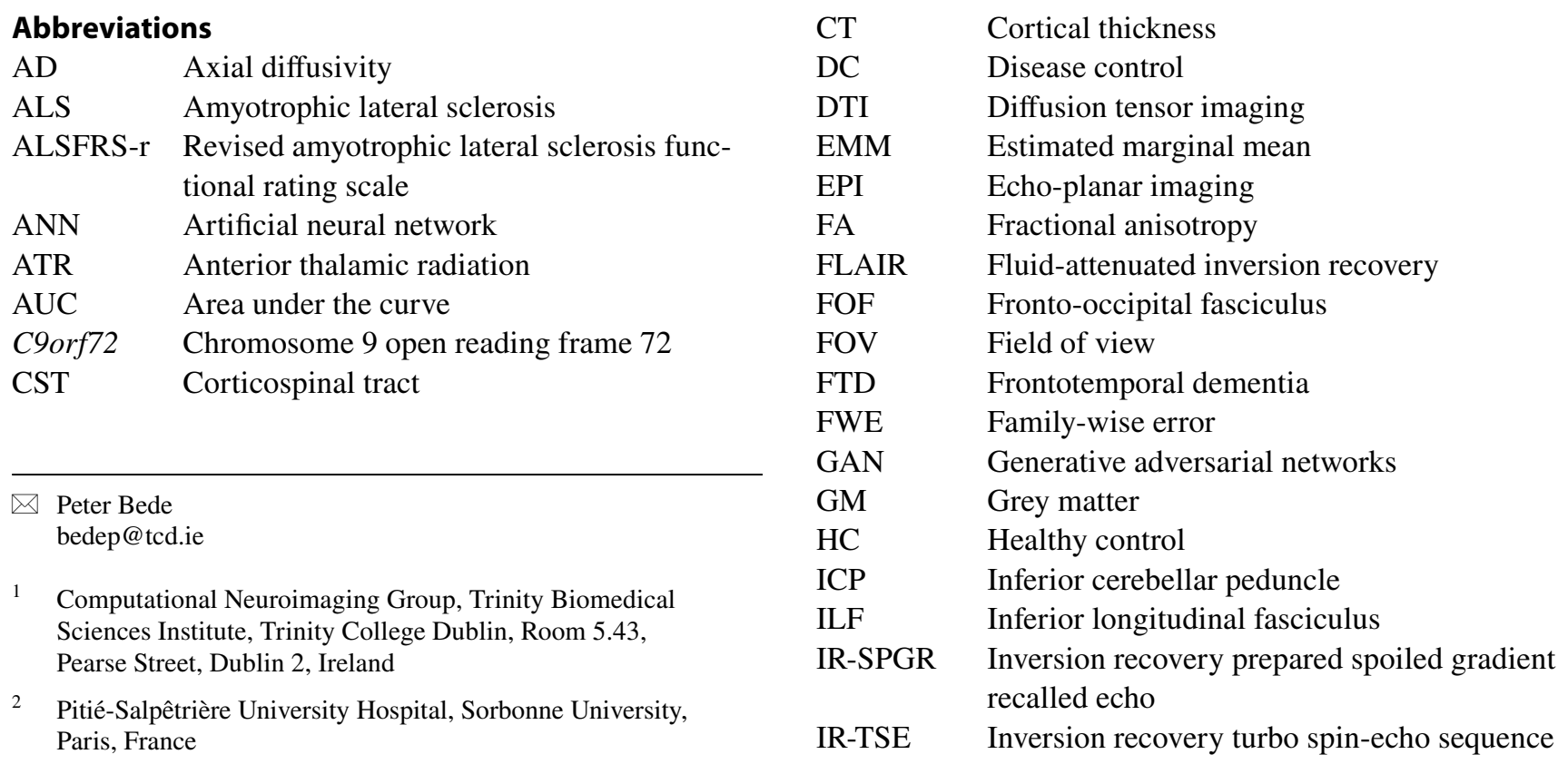




$\begin{array}{ll}\text { IVIG } & \text { Intravenous immunoglobulin } \\ \text { LMN } & \text { Lower motor neuron } \\ \text { LO } & \text { Lateral occipital } \\ \text { Lt } & \text { Left } \\ \text { MCP } & \text { Middle cerebellar peduncle } \\ \text { MD } & \text { Mean diffusivity } \\ \text { ML } & \text { Machine learning } \\ \text { MLe } & \text { Medial lemniscus } \\ \text { MND } & \text { Motor neuron disease } \\ \text { MNI152 } & \text { Montreal Neurological Institute } 152 \text { standard } \\ & \text { space } \\ \text { MPM } & \text { Multilayer perceptron model } \\ \text { NISALS } & \text { Neuroimaging Society in ALS } \\ \text { PBA } & \text { Pseudobulbar affect } \\ \text { PCC } & \text { Pathological crying and laughing } \\ \text { PMC } & \text { Primary motor cortex } \\ \text { QC } & \text { Quality control } \\ \text { RD } & \text { Radial diffusivity } \\ \text { ROC } & \text { Receiver-operating characteristic curve } \\ \text { ROI } & \text { Region of interest } \\ \text { Rt } & \text { Right } \\ \text { SCP } & \text { Superior cerebellar peduncle } \\ \text { SD } & \text { Standard deviation } \\ \text { SE-EPI } & \text { Spin-echo echo planar imaging } \\ \text { SENSE } & \text { Sensitivity encoding } \\ \text { SLF } & \text { Superior longitudinal fasciculus } \\ \text { SPIR } & \text { Spectral presaturation with inversion } \\ & \text { recovery } \\ \text { T1w } & \text { T1-weighted imaging } \\ \text { TBSS } & \text { Tract-based spatial statistics } \\ \text { TE } & \text { Echo time } \\ \text { TFCE } & \text { Threshold-free cluster enhancement } \\ \text { TI } & \text { Inversion time } \\ \text { TIV } & \text { Total intracranial volume } \\ \text { TR } & \text { Repetition time } \\ \text { UF } & \text { Uncinate fasciculus } \\ \text { UMN } & \text { Upper motor neuron } \\ \text { WM } & \text { White matter } \\ & \end{array}$

\section{Introduction}

Diagnostic delay in neurodegenerative conditions has a considerable literature. In ALS, the average interval from symptom onset to definite diagnosis is around 12 months [1, 2]. Patients often describe insidious symptom onset many months before medical advice is sought. The key milestones of the diagnostic journey in ALS include symptom manifestation, visit to a general practitioner, review in a general neurology clinic, diagnostic investigations, and assessment in a tertiary referral centre to confirm a suspected diagnosis [3-6]. The constellation of initial symptoms may be confounded by comorbid conditions, and misdiagnoses in the initial phase of ALS are not uncommon. The implications of diagnostic delay are considerable as it may delay recruitment into clinical trials, may have ramifications for genetic counselling, may increase the risk of misdiagnoses or potentially lead to unnecessary medical or surgical interventions such as spinal laminectomies, carpal tunnel surgery, and intravenous immunoglobulin (IVIg) treatment [1]. Recent imaging studies have revealed that by the time the diagnosis is confirmed, significant neurodegenerative changes have already occurred [7], limiting the potential of putative neuroprotective medications. Recent evidence also suggests that considerable presymptomatic disease burden can be readily detected long before symptom manifestation [8-11]. These observations would suggest that, the optimal window for clinical trials is not well into the 'post-diagnostic' phase of the disease, when widespread cerebral and spinal cord degeneration can already be detected, but as early as the diagnostic likelihood or mutation status permit. The role of neuroimaging in ALS has been extensively discussed [12], but the literature is dominated by papers describing grouplevel, phenotype- or genotype-associated imaging traits [13]. Various research consortia have invested considerable effort to increase cohort numbers, pool data from multiple centres to perform well-powered analyses and report radiological patterns representative of a particular phenotype [14]. The characterisation of stereotyped 'signatures' is academically interesting $[12,15]$, but the practical demands of clinical practice are markedly different [16]. As opposed to the scholarly pursuit of group-level descriptions, the priority of clinical neurology is the precision classification of a specific, single patient into diagnostic, phenotypic and prognostic categories through the quantitative interpretation of their biomarker profile. Relatively few studies have focussed on the classification of individual patient imaging data in ALS [17, 18]. A variety of innovative approaches have been explored [19] spanning from z-score based approaches, through support vector machine frameworks, discriminant function analyses, to regression models, with varying degree of classification accuracy [16, 20-24]. Several studies have reported excellent 'area under the curve' (AUC) values with reference to the discriminatory potential of a specific measure between patients and healthy controls, but binary classification into 'ALS' versus 'healthy' does not mirror real-life diagnostic dilemmas. In the clinical setting, the distinction between 'ALS' and 'healthy' is seldom challenging; instead, the dilemma is typically whether subtle clinical changes represent incipient ALS or rather, the harbinger of an alternative neurodegenerative condition. Another common shortcoming of classification studies is the a priori selection of anatomical regions, often referred to as 'regions of interest' (ROIs) which are known to be affected in ALS, rather than performing formal feature selection analyses or ranking variables based on their discriminatory potential. 
Finally, few studies have narrowed their analyses to a cohort of patients in their peri-diagnostic phase, which seems indispensable to scrutinise and validate proposed frameworks. The classification of cases with marked disability and long disease duration reveals relatively little about the efficacy of a specific model architecture. Accordingly, the objective of this study is the development of an observer-independent, multiclass (three-way) classification protocol to categorise multiparametric imaging data of a large cohort of subjects consisting of patients with amyotrophic lateral sclerosis (ALS), healthy controls (HC) and disease controls (DC). An additional objective of the study is to evaluate and rank the importance of imaging measures and anatomical foci for further model optimisation, and to test a proposed classification framework on subjects in their peri-diagnostic phase.

\section{Methods}

\section{Participants}

A total of 378 participants, 214 patients with ALS ('ALS'), 37 disease controls ('DC') with a non-ALS neurodegenerative diagnosis and 127 healthy controls ('HC') were included in a prospective, single-centre imaging study. All participants gave informed consent in accordance with the Ethics Approval of this research project (Beaumont Hospital, Dublin, Ireland). Exclusion criteria included prior cerebrovascular events, known traumatic brain injury, comorbid neoplastic, paraneoplastic or neuroinflammatory diagnoses. Participating ALS patients were diagnosed according to the revised El Escorial criteria. Disease controls consisted of patients with FTD and were diagnosed based on the Rascovsky criteria. Participating patients had a uniform neurological assessment and key variables, such as disability scores, interval from diagnosis to scan, and handedness were recorded.

\section{Magnetic resonance imaging}

A standardised imaging protocol was implemented on a 3 Tesla Philips Achieva Magnetic resonance (MR) platform. A 3D Inversion Recovery prepared Spoiled Gradient Recalled echo (IR-SPGR) sequence was utilised to acquire T1-weighted (T1w) images with a field-of-view (FOV) of $256 \times 256 \times 160 \mathrm{~mm}$, flip angle $=8^{\circ}$, spatial resolution of $1 \mathrm{~mm}^{3}$, SENSE factor $=1.5, \mathrm{TR} / \mathrm{TE}=8.5 / 3.9 \mathrm{~ms}$, $\mathrm{TI}=1060 \mathrm{~ms}$. A spin-echo echo planar imaging (SEEPI) pulse sequence was used to acquire diffusion tensor images (DTI) using a 32-direction Stejskal-Tanner diffusion encoding scheme; FOV $=245 \times 245 \times 150 \mathrm{~mm}, 60$ slices with no interslice gap, spatial resolution $=2.5 \mathrm{~mm}^{3}$, TR/ $\mathrm{TE}=7639 / 59 \mathrm{~ms}$, SENSE factor $=2.5, b$ values $=0,1100 \mathrm{~s} /$ $\mathrm{mm}^{2}$, dynamic stabilisation and spectral presaturation with inversion recovery (SPIR) fat suppression. To assess for comorbid inflammatory or vascular pathologies, fluid-attenuated inversion recovery (FLAIR) images were also acquired from each subject. An Inversion Recovery Turbo Spin Echo (IR-TSE) sequence was used for FLAIR imaging. Data were acquired in axial orientation: FOV $=230 \times 183 \times 150 \mathrm{~mm}$, spatial resolution $=0.65 \times 0.87 \times 4 \mathrm{~mm}, 30$ slices with $1 \mathrm{~mm}$ gap, TR/TE $=11,000 / 125 \mathrm{~ms}, \mathrm{TI}=2800 \mathrm{~ms}, 120^{\circ}$ refocusing pulse, with flow compensation and motion smoothing and a saturation slab covering the neck region.

\section{Imaging framework}

Initial quality control steps included radiological review for incidental pathological findings, assessment for movement artefacts, and evaluation of white matter abnormalities on FLAIR. Following standardised pre-processing steps (described below), 28 volumetric metrics, 68 cortical thickness values and 120 white matter indices were uniformly retrieved from each subject's imaging data; a total of 216 imaging measures were then appraised in each participant. These data were systematically analysed in post-hoc statistics.

\section{Volume metrics}

The standard anatomical reconstruction pipeline of the FreeSurfer image analysis suite [25], 'recon-all' was implemented, including non-parametric non-uniform intensity normalisation, affine registration to the MNI305 atlas, intensity normalisation, skull striping, automatic subcortical segmentation, linear volumetric registration, neck removal, tessellation of the grey matter-white matter boundary, surface smoothing, inflation to minimise metric distortion, and automated topology correction [26]. To segment the brainstem into the medulla oblongata, pons and midbrain, a Bayesian segmentation algorithm was utilised, which relies on a probabilistic atlas of the brainstem and its neighbouring anatomical structures generated based on 49 scans [27]. The following 28 cerebral volume values were uniformly retrieved from each pre-processed T1-weighted dataset: (1) left cerebellar white matter volume, (2) left cerebellar cortex volume, (3) left thalamus volume, (4) left caudate volume, (5) left putamen volume, (6) left pallidum volume, (7) left hippocampus volume, (8) left amygdala volume, (9) left accumbens volume, (10) right cerebellar white matter volume, (11) right cerebellar cortex volume, (12) right thalamus volume, (13) right caudate volume, (14) right putamen volume, (15) right pallidum volume, (16) right hippocampus volume, (17) right amygdala volume, (18) right accumbens volume, (19) posterior corpus callosum volume, (20) middle corpus callosum volume, (21) central corpus callosum 
volume, (22) mid-anterior corpus callosum volume, (23) anterior corpus callosum volume, (24) medulla volume, (25) pons volume, (26) superior cerebellar peduncle volume, (27) midbrain volume, and (28) total intracranial volume. Volumetric values of individual subjects were converted as percentage of the subject's total intracranial volume (TIV) to account for individual TIV variations.

\section{Cortical thickness values}

Following pre-processing with 'recon-all', the labels of the Desikan-Killiany atlas were utilised to retrieve average cortical thickness values [20] from 34 cortical regions in the left and right cerebral hemispheres; (1) banks superior temporal sulcus, (2) caudal anterior-cingulate cortex, (3) caudal middle frontal gyrus, (4) cuneus cortex, (5) entorhinal cortex, (6) frontal pole, (7) fusiform gyrus, (8) inferior parietal cortex, (9) inferior temporal gyrus, (10) insula, (11) isthmus-cingulate cortex, (12) lateral occipital cortex, (13) lateral orbitofrontal cortex, (14) lingual gyrus, (15) medial orbital frontal cortex, (16) middle temporal gyrus, (17) parahippocampal gyrus, (18) paracentral lobule, (19) pars opercularis, (20) pars orbitalis, (21) pars triangularis, (22) pericalcarine cortex, (23) postcentral gyrus (24) posterior-cingulate cortex, (25) precentral gyrus, (26) precuneus cortex, (27) rostral anterior-cingulate cortex, (28) rostral middle frontal gyrus, (29) superior frontal gyrus, (30) superior parietal cortex, (31) superior temporal gyrus, (32) supramarginal gyrus, (33) temporal pole, and (34) transverse temporal cortex.

\section{White matter indices}

Pre-processing of diffusion tensor data were implemented using in FMRIB's software library. Raw DTI data first underwent eddy current corrections and skull removal; a tensor model was then fitted to generate maps of axial diffusivity (AD), fractional anisotropy (FA), mean diffusivity (MD), and radial diffusivity (RD). FMRIB's software library's tract-based statistics (TBSS) module was utilised for non-linear registration and skeletonisation of individual DTI images. A mean FA mask was created and each subject's individual AD, FA, MD and RD images were merged into 4-dimensional (4D) AD, FA, MD and RD image files. The study-specific white matter skeleton was masked by atlas-defined labels for the following 30 white matter regions of interests in MNI space: (1) left anterior thalamic radiation, (2) right anterior thalamic radiation, (3) left cerebellar white matter skeleton averaged, (4) right cerebellar white matter skeleton averaged, (5) left cingulum, (6) right cingulum, (7) left corticospinal tract, (8) right corticospinal tract, (9) left external capsule, (10) right external capsule, (11) forceps major, (12) forceps minor, (13) fornix, (14) left inferior cerebellar peduncle, (15) right inferior cerebellar peduncle, (16) left inferior fronto-occipital fasciculus, (17) right inferior fronto-occipital fasciculus, (18) left inferior longitudinal fasciculus, (19) right inferior longitudinal fasciculus, (20) left medial lemniscus, (21) right medial lemniscus, (22) middle cerebellar peduncle, (23) left posterior thalamic radiation, (24) right posterior thalamic radiation, (25) left superior cerebellar peduncle, (26) right superior cerebellar peduncle, (27) left superior longitudinal fasciculus, (28) right superior longitudinal fasciculus, (29) left uncinate fasciculus, and (30) right uncinate fasciculus. The labels of the standard-space ICBM-DTI-81 white matter atlas [28, 29] were utilised to create masks for the cerebellar peduncles, medial lemniscus, external capsule and posterior thalamic radiation. Labels of the JHU white matter tractography atlas $[30,31]$ were used to generate masks for the forceps major and minor, anterior thalamic radiation, uncinate, superior and inferior longitudinal fasciculi, cingulum, corticospinal tracts, inferior fronto-occipital fasciculi. The cerebellar label (label 2) of the MNI probabilistic atlas [32,33] was used to generate a mask for averaged cerebellar diffusivity estimation. The FMRIB fornix template [34] was used to mask the study-specific white matter skeleton in MNI space. Four diffusivity metrics (AD, FA, MD, RD) were retrieved from 30 white matter regions in each subject, resulting in a white matter panel of 120 values.

\section{Statistical analyses}

An artificial neural network framework, a multilayer perceptron model was implemented with hyperbolic tangent as the hidden layer activation function. The diagnosis (ALS, HC, DC) was set as dependent variable, and the retrieved imaging measures as covariates. Imaging metrics were rescaled by standardisation; $(x-$ mean $) / \mathrm{s}$. The model architecture included one hidden layer with 6 units. Data were partitioned into a training sample $(68 \%)$ and testing sample (32\%). A batch-type training approach was utilised with a gradient descent optimisation algorithm; initial learning rate: 0.4 , momentum: 0.9 , interval centre: 0 , interval offset: \pm 0.5 . Using the above model architecture, the following outputs were generated; synaptic weights, classification results, ROC curves, and AUC values. An independent variable importance analysis was also performed to rank the relevance of imaging metrics in determining group membership. To visually represent the accuracy of diagnostic classification, the predicted pseudo-probability of each diagnostic group was plotted in a bar chart. Based on the feature importance hierarchy, a streamlined classification model was tested using only the 20 most important imaging variables. Finally, to further scrutinise the classification framework, the model was tested on a subset of patients in their peri-diagnosis phase, who were scanned within 6 weeks of their formal diagnosis. 


\section{Results}

The three groups, ALS $(n=214$, age: $60.97 \pm 11.92$, 140 male, 202 right handed), healthy controls $(n=127$, age: $59.29 \pm 10.95,59$ male, 112 right handed) and disease controls $(n=37$, age: $62.4 \pm 7.9,20$ male, 34 right handed) were matched for age $(p=0.23)$ and handedness $(p=0.12)$, but not for sex $(p=0.002)$. Of the 378 datasets, $256(67.7 \%)$ was included in the training sample and $122(32.3 \%)$ in the testing sample. Cross-entropy error was 73.49 in the training sample and 85.03 in the testing sample; incorrect predications were $10.9 \%$ in the training sample and $24.6 \%$ in the testing sample. Classification summary is presented in Table 1A. The predicted pseudo-probability of diagnosis in each cohort (confirmed

Table 1 Classification outcomes in the training and testing samples using A, all imaging features B, the 20 most important variables only and $\mathrm{C}$, white matter diffusivity metrics alone

\begin{tabular}{|c|c|c|c|c|c|}
\hline \multirow[t]{2}{*}{ Sample } & \multirow[t]{2}{*}{ Observed } & \multicolumn{4}{|c|}{ Predicted } \\
\hline & & ALS & DC & $\mathrm{HC}$ & $\%$ Correct \\
\hline \multicolumn{6}{|c|}{ (A) All features included } \\
\hline \multirow[t]{4}{*}{ Training } & ALS & 140 & 2 & 9 & 92.7 \\
\hline & DC & 3 & 19 & 2 & 79.2 \\
\hline & $\mathrm{HC}$ & 11 & 1 & 69 & 85.2 \\
\hline & Overall percent & $60.2 \%$ & $8.6 \%$ & $31.3 \%$ & 89.1 \\
\hline \multirow[t]{4}{*}{ Testing } & ALS & 52 & 6 & 5 & 82.5 \\
\hline & $\mathrm{DC}$ & 2 & 9 & 2 & 69.2 \\
\hline & $\mathrm{HC}$ & 14 & 1 & 31 & 67.4 \\
\hline & Overall percent & $55.7 \%$ & $13.1 \%$ & $31.1 \%$ & 75.4 \\
\hline \multicolumn{6}{|c|}{ (B) 20 core features included } \\
\hline \multirow[t]{4}{*}{ Training } & ALS & 126 & 1 & 32 & 79.2 \\
\hline & DC & 3 & 23 & 1 & 85.2 \\
\hline & $\mathrm{HC}$ & 24 & 0 & 63 & 72.4 \\
\hline & Overall percent & $56.0 \%$ & $8.8 \%$ & $35.2 \%$ & 77.7 \\
\hline \multirow[t]{4}{*}{ Testing } & ALS & 46 & 0 & 9 & 83.6 \\
\hline & DC & 2 & 7 & 1 & 70.0 \\
\hline & $\mathrm{HC}$ & 15 & 0 & 25 & 62.5 \\
\hline & Overall percent & $60.0 \%$ & $6.7 \%$ & $33.3 \%$ & 74.3 \\
\hline \multicolumn{6}{|c|}{ (C) Only white matter metrics included } \\
\hline \multirow[t]{4}{*}{ Training } & ALS & 128 & 1 & 18 & 87.1 \\
\hline & DC & 0 & 24 & 0 & 100.0 \\
\hline & $\mathrm{HC}$ & 16 & 0 & 70 & 81.4 \\
\hline & Overall percent & $56.0 \%$ & $9.7 \%$ & $34.2 \%$ & 86.4 \\
\hline \multirow[t]{4}{*}{ Testing } & ALS & 53 & 3 & 11 & 79.1 \\
\hline & $\mathrm{DC}$ & 0 & 12 & 1 & 92.3 \\
\hline & $\mathrm{HC}$ & 11 & 0 & 30 & 73.2 \\
\hline & Overall percent & $52.9 \%$ & $12.4 \%$ & $34.7 \%$ & 78.5 \\
\hline
\end{tabular}

$A L S$ amyotrophic lateral sclerosis, $D C$ disease control, $H C$ healthy control

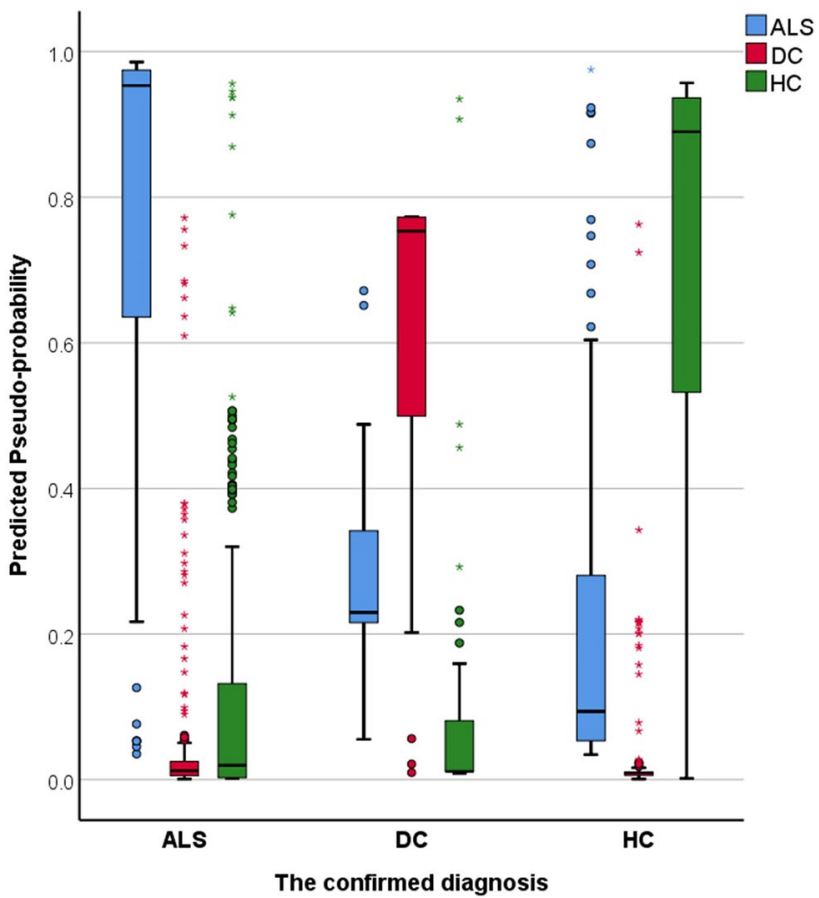

Fig. 1 The predicted pseudo-probability profiles of subjects with an established diagnosis

diagnosis) is presented in Fig. 1. Receiver-operating characteristic (ROC) curves are presented in Fig. 2A. Area under the curve values were 0.930 for ALS, 0.958 for disease controls, and 0.931 for healthy controls relying on all input imaging variables. The normalised importance of the 20 most relevant imaging variables in predicting group membership is shown in Fig. 3 with their corresponding importance value. The ranked normalised importance of the 50 most relevant imaging metrics is presented in Table 2. The classification analyses were re-run with the 20 most important imaging metrics identified by the explorative analyses. Relying on only 20 imaging features, the classification accuracy was evaluated again (Table 1B). Area under the curve values based on only 20 core imaging features (Fig. 2B) were 0.835 for ALS, 0.990 for DC, and 0.842 for healthy controls. As 19 white matter metrics were ranked among the 20 most important imaging features (Fig. 3) and the vast majority (92\%) of imaging metrics among the 50 diagnostically relevant variables (Table 2) were diffusivity metrics, a final post hoc analysis was conducted where only white matter diffusivity indices were included as covariates in the perceptron model; all 30 tracts and all four diffusivity metrics (120 variables in total). Area under the receiver-operating characteristic curves generated based on white matter features alone (Fig. 2C) were 0.907 for ALS, 0.979 for DC, and 0.911 for healthy controls. Classification outcomes using white measures alone are presented in Table 1C. 

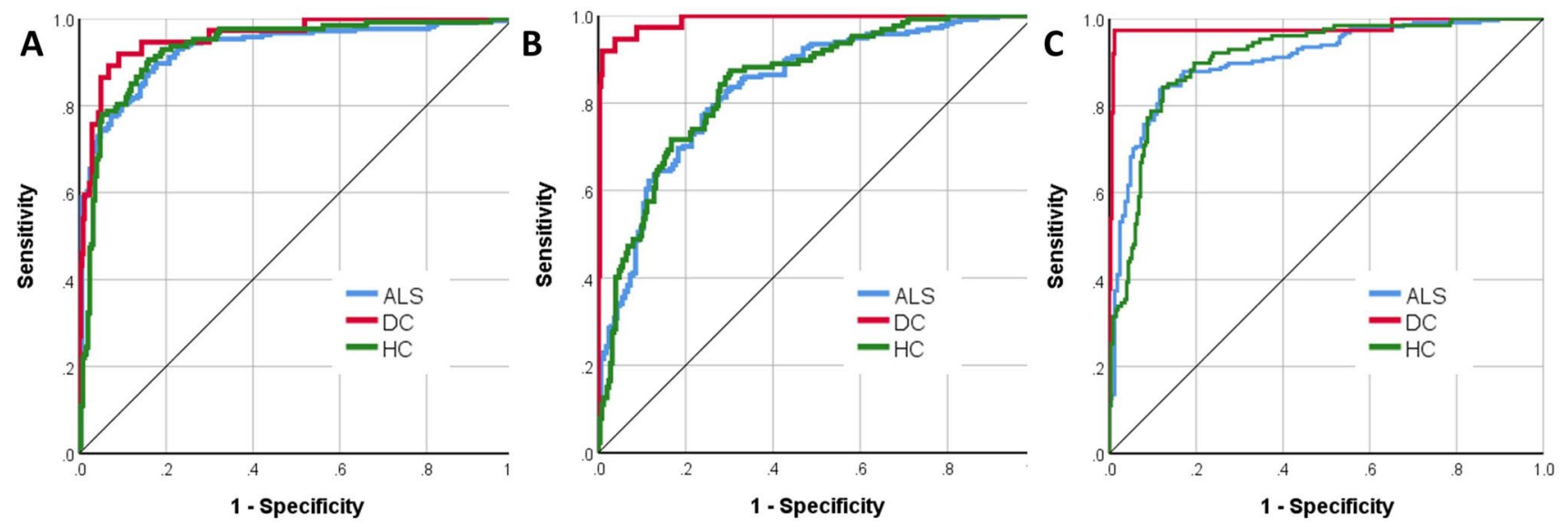

Fig. 2 Receiver-operating characteristic curve of patients with ALS, disease controls (DC) and healthy controls (HC) using all imaging features (A-left), the 20 most important variables only $(\mathbf{B}$ - middle) and white matter diffusivity metrics alone (C-right)

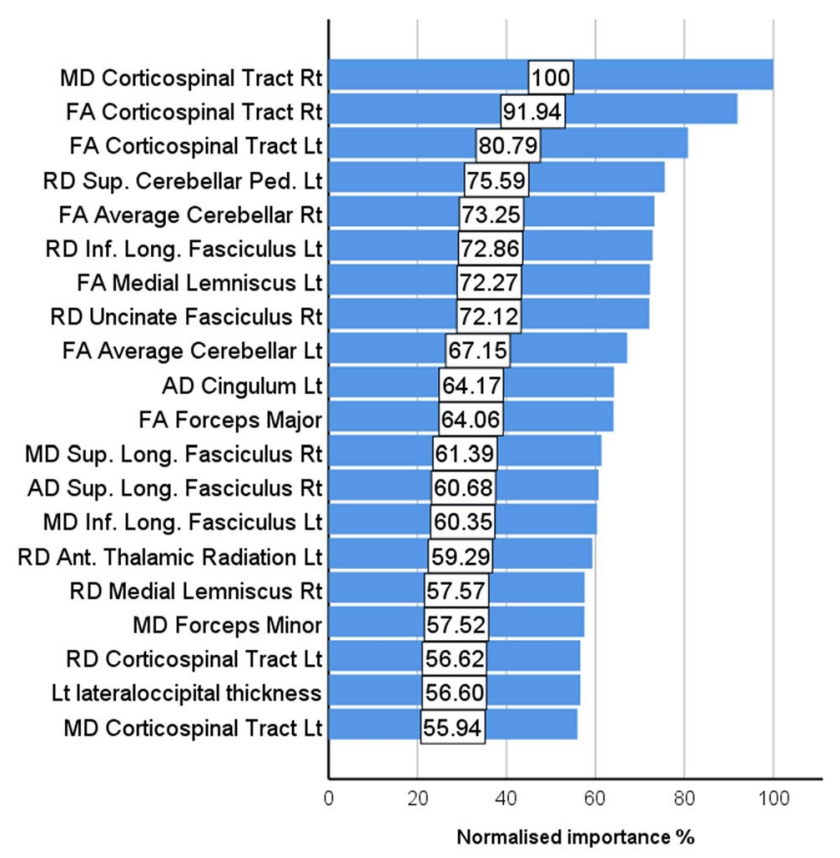

Fig. 3 The hierarchy of normalised variable importance

To scrutinise the validity of this classification strategy, the model was tested on a subset of patients $(n=119)$ who were scanned within 6 weeks of their diagnoses ("peri-diagnosis cohort'). Using all imaging features AUC was 0.915 for ALS, 0.979 for DC and 0.929 for HC. Using the 20 most important imaging features alone, AUC was 0.822 for ALS, 0.958 for DC and 0.853 for HC. Using all WM metrics but no GM measures, AUC was 0.914 for ALS, 0.981 for DC and 0.92 for HC. Classification outcomes in the 'peri-diagnosis' cohort are presented in Table 3. Pseudo-probability profiles in the peri-diagnostic phase using all imaging features are presented in Fig. 4 and the three ROC curves are shown in Fig. 5. Model architecture is presented in Fig. 6 with 20 input variables.

\section{Discussion}

Our data indicate that quantitative imaging aids diagnostic classification and the systematic assessment of key anatomical regions may not only help to distinguish ALS from healthy controls, but also discriminates it from other neurodegenerative conditions. The presented framework operates in an observer-independent fashion and receiver-operating characteristic curves indicate excellent sensitivity/specificity profiles. In addition to the classification accuracy of the multilayer perceptron model utilised, the ranking of imaging features with respect to categorisation relevance offers valuable insights for the streamlining and optimisation of future models.

The utility of a variety of supervised and unsupervised machine-learning approaches have been explored in ALS, including support vector machines, regressionbased approaches, random forests, discriminant function analyses, dimension reduction frameworks, but these are seldom applied to imaging data $[17,35,36]$ due to challenges associated with MRI scanning, quality control, preprocessing, data acquisition costs and data harmonisation. Advanced neural network architectures have been successfully trialled in other conditions, including multilayer 'deep-learning' learning models and generative adversarial networks (GAN) [37-40]. The development of automated diagnostic frameworks based on radiology data in ALS are hampered by the scarcity of large, uniformly acquired training data sets. While the acquisition and recording of epidemiology data and clinical measures can be relatively easily 
Table 2 The importance and normalised importance of the 50 most relevant imaging variables in predicting group membership

\begin{tabular}{|c|c|c|c|}
\hline Rank & Imaging metric & Importance & $\begin{array}{l}\text { Normalised } \\
\text { importance }(\%)\end{array}$ \\
\hline 1. & MD Corticospinal Tract Rt & 0.014 & 100.0 \\
\hline 2. & FA Corticospinal Tract Rt & 0.013 & 91.9 \\
\hline 3. & FA Corticospinal Tract Lt & 0.011 & 80.8 \\
\hline 4. & RD Sup Cerebellar Ped Lt & 0.010 & 75.6 \\
\hline 5. & FA Average Cerebellar Rt & 0.010 & 73.3 \\
\hline 6. & RD Inf. Longitudinal_Fasciculus Lt & 0.010 & 72.9 \\
\hline 7. & FA Medial Lemniscus Lt & 0.010 & 72.3 \\
\hline 8. & RD Uncinate Fasciculus Rt & 0.010 & 72.1 \\
\hline 9. & FA Average Cerebellar Lt & 0.009 & 67.1 \\
\hline 10. & AD Cingulum Lt & 0.009 & 64.2 \\
\hline 11. & FA Forceps Major & 0.009 & 64.1 \\
\hline 12. & MD Sup. Longitudinal Fasciculus Rt & 0.008 & 61.4 \\
\hline 13. & AD Sup. Longitudinal Fasciculus Rt & 0.008 & 60.7 \\
\hline 14. & MD Inf. Longitudinal Fasciculus Lt & 0.008 & 60.4 \\
\hline 15 & RD Anterior Thalamic Radiation Lt & 0.008 & 59.3 \\
\hline 16. & RD Medial Lemniscus Rt & 0.008 & 57.6 \\
\hline 17. & MD Forceps Minor & 0.008 & 57.5 \\
\hline 18. & RD Corticospinal Tract Lt & 0.008 & 56.6 \\
\hline 19. & Lt lateraloccipital thickness & 0.008 & 56.6 \\
\hline 20. & MD Corticospinal Tract Lt & 0.008 & 55.9 \\
\hline 21. & RD Medial Lemniscus Lt & 0.008 & 55.6 \\
\hline 22. & AD External Capsule Rt & 0.008 & 54.6 \\
\hline 23. & FA Uncinate Fasciculus Rt & 0.007 & 54.2 \\
\hline 24. & RD Sup. Cerebellar Ped Rt & 0.007 & 54.0 \\
\hline 25. & Lt posteriorcingulate thickness & 0.007 & 53.7 \\
\hline 26. & AD Forceps Minor & 0.007 & 53.6 \\
\hline 27. & RD Anterior Thalamic Radiation Rt & 0.007 & 53.5 \\
\hline 28. & FA Inf. Cerebellar Peduncle Rt & 0.007 & 53.1 \\
\hline 29. & FA Inf. Longitudinal Fasciculus Rt & 0.007 & 52.4 \\
\hline 30. & Lt precentral thickness & 0.007 & 51.1 \\
\hline 31. & RD Average Cerebellar Lt & 0.007 & 51.0 \\
\hline 32. & RD Inf. Cerebellar Peduncle Lt & 0.007 & 51.0 \\
\hline 33. & MD Middle Cerebellar Peduncle & 0.007 & 50.7 \\
\hline 34. & RD Inf. Cerebellar Peduncle Rt & 0.007 & 50.1 \\
\hline 35. & FA Inf. Cerebellar Peduncle Lt & 0.007 & 49.9 \\
\hline 36. & MD Post. Thalamic Radiation Rt & 0.007 & 49.8 \\
\hline 37. & MD Inf. Fronto-Occipital Fasciculus Rt & 0.007 & 49.7 \\
\hline 38. & FA Inf. Longitudinal Fasciculus Lt & 0.007 & 49.3 \\
\hline 39. & FA Sup. Cerebellar Peduncle Lt & 0.007 & 48.8 \\
\hline 40. & MD Medial Lemniscus Lt & 0.007 & 48.3 \\
\hline 41. & Lt Caudal middle frontal thickness & 0.007 & 48.2 \\
\hline 42. & AD Medial Lemniscus Rt & 0.007 & 47.4 \\
\hline 43. & Left-Amygdala Volume $\%$ & 0.007 & 47.1 \\
\hline 44. & RD Middle Cerebellar Peduncle & 0.007 & 47.0 \\
\hline 45. & MD Anterior Thalamic Radiation Rt & 0.006 & 46.8 \\
\hline 46. & FA Uncinate Fasciculus Lt & 0.006 & 45.7 \\
\hline 47. & FA Medial Lemniscus Rt & 0.006 & 45.4 \\
\hline 48. & RD Forceps Major & 0.006 & 45.3 \\
\hline 49. & RD Forceps Minor & 0.006 & 45.0 \\
\hline 50. & MD External Capsule Rt & 0.006 & 45.0 \\
\hline
\end{tabular}

$A D$ axial diffusivity, $A L S$ amyotrophic lateral sclerosis, $D C$ disease control, $F A$ fractional anisotropy, $H C$ healthy control, $L t$ left, $M D$ mean diffusivity, $R D$ radial diffusivity, $R t$ right 
Table 3 Classification outcomes in the peri-diagnostic phase in the training and testing samples using A, all imaging features B, the 20 most important variables only and $\mathrm{C}$, white matter diffusivity metrics alone

\begin{tabular}{|c|c|c|c|c|c|}
\hline \multirow[t]{2}{*}{ Sample } & \multirow[t]{2}{*}{ Observed } & \multicolumn{4}{|c|}{ Predicted } \\
\hline & & ALS & $\mathrm{DC}$ & $\mathrm{HC}$ & $\%$ Correct \\
\hline \multicolumn{6}{|c|}{ (A) All features included } \\
\hline \multirow[t]{4}{*}{ Training } & ALS & 76 & 5 & 9 & 84.4 \\
\hline & DC & 1 & 19 & 3 & 82.6 \\
\hline & $\mathrm{HC}$ & 15 & 1 & 65 & 80.2 \\
\hline & Overall percent & $47.4 \%$ & $12.9 \%$ & $39.7 \%$ & 82.5 \\
\hline \multirow[t]{4}{*}{ Testing } & ALS & 23 & 0 & 6 & 79.3 \\
\hline & $\mathrm{DC}$ & 2 & 11 & 1 & 78.6 \\
\hline & $\mathrm{HC}$ & 11 & 0 & 35 & 76.1 \\
\hline & Overall percent & $40.4 \%$ & $12.4 \%$ & $47.2 \%$ & 77.5 \\
\hline \multicolumn{6}{|c|}{ (B) 20 features included } \\
\hline \multirow[t]{4}{*}{ Training } & ALS & 58 & 3 & 24 & 68.2 \\
\hline & $\mathrm{DC}$ & 3 & 22 & 1 & 84.6 \\
\hline & $\mathrm{HC}$ & 15 & 1 & 68 & 81.0 \\
\hline & Overall percent & $39.0 \%$ & $13.3 \%$ & $47.7 \%$ & 75.9 \\
\hline \multirow[t]{4}{*}{ Testing } & ALS & 26 & 4 & 4 & 76.5 \\
\hline & $\mathrm{DC}$ & 1 & 6 & 4 & 54.5 \\
\hline & $\mathrm{HC}$ & 7 & 0 & 36 & 83.7 \\
\hline & Overall percent & $38.6 \%$ & $11.4 \%$ & $50.0 \%$ & 77.3 \\
\hline \multicolumn{6}{|c|}{ (C) Only white matter metrics included } \\
\hline \multirow[t]{4}{*}{ Training } & ALS & 62 & 3 & 13 & 79.5 \\
\hline & $\mathrm{DC}$ & 1 & 24 & 0 & 96.0 \\
\hline & $\mathrm{HC}$ & 12 & 1 & 75 & 85.2 \\
\hline & Overall percent & $39.3 \%$ & $14.7 \%$ & $46.1 \%$ & 84.3 \\
\hline \multirow[t]{4}{*}{ Testing } & ALS & 30 & 2 & 9 & 73.2 \\
\hline & $\mathrm{DC}$ & 2 & 9 & 1 & 75.0 \\
\hline & $\mathrm{HC}$ & 5 & 1 & 33 & 84.6 \\
\hline & Overall percent & $40.2 \%$ & $13.0 \%$ & $46.7 \%$ & 78.3 \\
\hline
\end{tabular}

$A L S$ amyotrophic lateral sclerosis, $D C$ disease control, $H C$ healthy control

harmonised, imaging data harmonisation requires considerable investment.

Our study consisted of an exploratory arm, where imaging metrics from the entire cerebrum were incorporated, without the a priori selection of anatomic regions considered relevant based on published imaging or post mortem evidence. While the predilection of disease burden to the corticospinal tracts, precentral gyrus and brainstem is well established, our strategy centred on the indiscriminate interrogation of imaging variables from across the entire cerebrum. To develop a truly observer-independent pipeline, individual imaging data were spatially co-registered to standard space, and only validated atlases were utilised to retrieve integrity variables from a range of anatomical regions. Cortical and subcortical, grey matter and white matter, supratentorial and infratentorial, left and right hemisphere structures

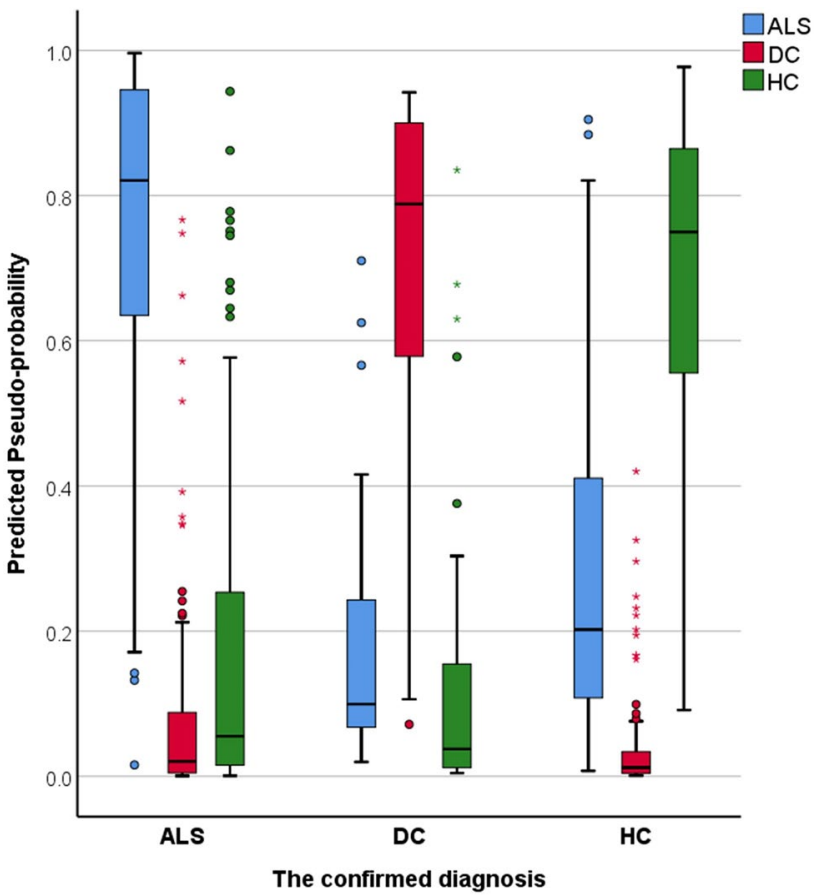

Fig. 4 The predicted pseudo-probability profiles of ALS patients around the time of their diagnosis ( $<6$ weeks), disease controls (DC) and healthy controls (HC)

were uniformly evaluated without prioritising potentially discriminatory anatomical regions a priori.

The ranking of variable importance revealed interesting trends. By large, integrity metrics of white matter regions discriminated the three groups better than grey matter measures. This is in line with previous observations that white matter degeneration is a relatively early feature of ALS [7, 41], while GM changes are less consistent, and may only become readily detectable in the later stages of the disease. A practical ramification of the recognition of the superior discriminatory power of white matter measures is that diffusion tensor protocols should be routinely incorporated into clinical and pharmacological trials protocols as opposed to only relying on T1-weighted, FLAIR and T2-weighted data sets which are classically used for clinical evaluation to rule out mimic conditions. Interestingly, there was only one grey matter variable among the first 20 diagnostically relevant imaging features and only 4 grey matter variables were ranked in the first 50 features. Our post hoc analyses also confirmed that excellent subject classification can be achieved relying on white matter measures alone (Figs. 2 and 5, Tables 1 and 3). These models provided accurate diagnostic classification without evaluating grey matter measures or volumes at all, and were solely based on measures derived from DTI. Furthermore, our results confirm the imperative of evaluating non-FA diffusivity measures. While FA is the most 

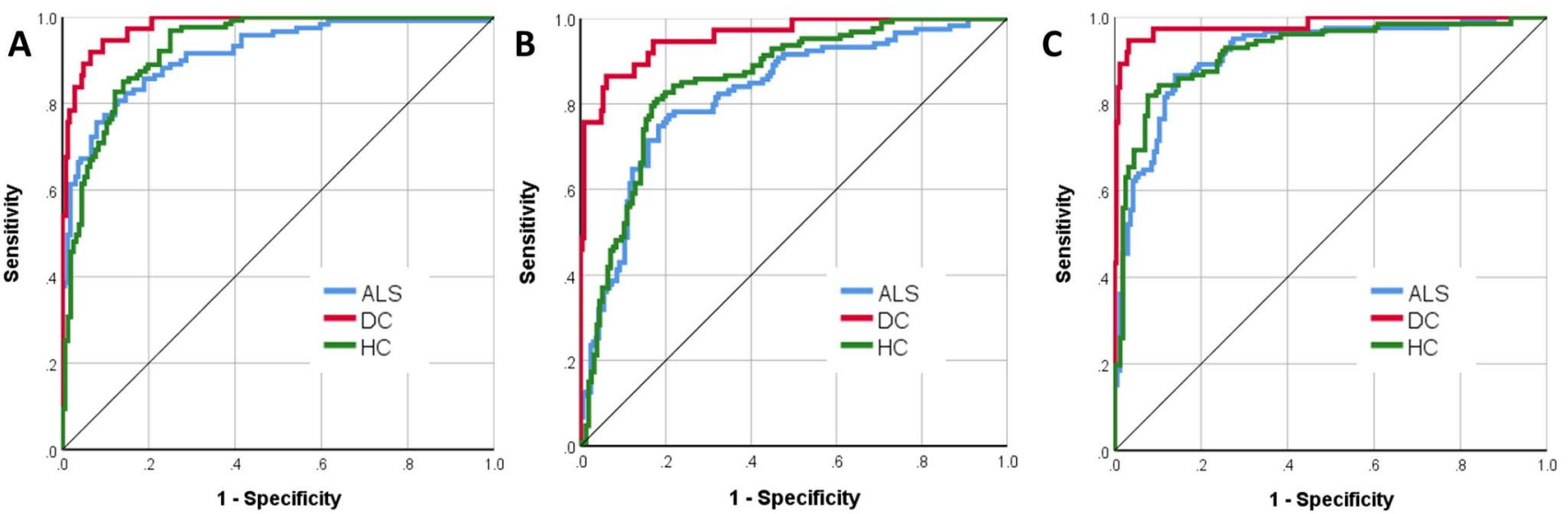

Fig. 5 Receiver-operating characteristic curve of patients with ALS around the time of their diagnosis ( $<6$ weeks), disease controls (DC) and healthy controls (HC) using all imaging features (A-left), the 20 most important variables only (B-middle) and white matter diffusivity metrics alone $(\mathbf{C}$-right $)$

commonly evaluated white matter metric in descriptive analyses, $\mathrm{RD}, \mathrm{MD}$, and $\mathrm{AD}$ proved to be equally important discriminatory variables in our models. The review of ranked discriminating variables (Table 2.) is not only interesting from the perspective of biophysical measures, but also from an anatomical standpoint. The relative importance of key ALS-associated brain regions such as corticospinal tracts and precentral gyrus is not surprising given the ample evidence of the pathognomonic involvement of these structures in ALS. Conversely, the indices of some brain regions, such as the brainstem, ranked relatively low in the hierarchy of feature importance despite their archetypal involvement in ALS [42]. The discriminatory relevance of external capsule integrity is also of interest as ALS studies overwhelmingly emphasise internal capsule alterations [43]. It is also noteworthy that multiple cerebellar measures are among the most important discriminatory features, including intra-cerebellar white matter diffusivity metrics, volumetric values as well as cerebellar peduncle integrity measures. The recognition that cerebellar degeneration is an important facet of ALS biology is not new, but regional cerebellar disease burden has only been recently characterised in detail [44-50]. Our findings highlight the practical importance of systematically evaluating infratentorial indices in ALS and not only focussing on supratentorial variables. Several long association tracts (ILF, FOF) were also listed among the first 50 discriminatory regions, which are likely to aid discrimination from the disease-control group [51]. Frontotemporal dementia is a genetically, molecularly and clinically heterogeneous group of conditions, and specific subtypes are associated with specific imaging signatures [52, 53]. Our study illustrates the relevance of assessing brain regions which are not classically affected in ALS [54]. These regions may be preferentially affected in other 
conditions therefore the interrogation of imaging metrics from these anatomical foci is invaluable in discriminating ALS from alternative diagnoses. More broadly, our results support the importance of exploring imaging data without a priori anatomical assumptions.

Our approach illustrates that a multitude of metrics may be readily incorporated into complex classification models across a variety of anatomical regions. These models may be potentially further expanded to include additional measures such as wet biomarkers, additional imaging metrics or clinical measures [55-64]. In this application only cerebral measures were evaluated, despite the potential of spinal metrics [65-68]. Similar frameworks could potentially be utilised for the discrimination of other MND phenotypes such as PLS, PMA or flail-arm syndrome [69-71].

This study is not without limitations. A three-way classification scheme was implemented with a single diseasecontrol group. The inclusion of a 'mimic' disease-control group would have been helpful to test the model further, but the definition of a true ALS mimic condition is contentious. Only total volumes of subcortical structures were explored as input variables in this study, even though the assessment of specific amygdalar nuclei, thalamic nuclei or hippocampal subfields may enhance the discrimination of ALS form other neurodegenerative conditions [72-75]. Moreover, our model only evaluated cerebral metrics, therefore LMN pathology is not accounted for and discrimination from LMN-predominant MNDs cannot be reliably assessed [76-82]. Additional validation of the model with presymptomatic mutation carriers would have tested the classification accuracy of the model further by evaluating subjects with limited disease burden [10,83]. Model overfitting to a particular training cohort is invariably a significant risk and this study is no exception. Notwithstanding these limitations, our results indicate that subjects may be accurately classified into a diagnostic cohort, healthy control, or diseases control categories based on imaging data alone.

\section{Conclusions}

The meaningful interpretation of singe-subject imaging data is an urgent priority of clinical neuroradiology. Group-level descriptive analyses offer valuable academic insights, but the practical demands of clinical neuroradiology and clinical trial applications require accurate single-subject classification based on a core set of quantitative markers.

Acknowledgements We are thankful for the participation of each patient and healthy control, and we also thank all the patients who expressed interest in this research study but were unable to participate for medical or logistical reasons. We also express our gratitude to the caregivers of patients for facilitating attendance at our neuroimaging centre. Without their generosity, this study would have not been possible.

Author contributions Drafting the manuscript: PB. Neuroimaging analyses: PB and AM. Conceptualisation of the study: PB and AM. Revision of the manuscript for intellectual content: $\mathrm{PB}, \mathrm{AM}$, and $\mathrm{OH}$.

Funding Open Access funding provided by the IReL Consortium. Professor Peter Bede and the Computational Neuroimaging Group are supported by the Health Research Board (HRB EIA-2017-019), the Irish Institute of Clinical Neuroscience (IICN), the Spastic Paraplegia Foundation (SPF), the EU Joint Programme-Neurodegenerative Disease Research (JPND), the Andrew Lydon scholarship, and the Iris O'Brien Foundation.

Availability of data and material Personal medical data are not publically available due to institutional data regulations.

Code availability Statistical procedures are described in the methods section of the manuscript.

\section{Declarations}

Conflicts of interest The authors have no competing interests to declare.

Ethics approval This study was approved by the Ethics (Medical Research) Committee-Beaumont Hospital, Dublin, Ireland.

Consent to participate All the participants had consented to participate.

Consent for publication All the participants had consented to publish these research findings.

Open Access This article is licensed under a Creative Commons Attribution 4.0 International License, which permits use, sharing, adaptation, distribution and reproduction in any medium or format, as long as you give appropriate credit to the original author(s) and the source, provide a link to the Creative Commons licence, and indicate if changes were made. The images or other third party material in this article are included in the article's Creative Commons licence, unless indicated otherwise in a credit line to the material. If material is not included in the article's Creative Commons licence and your intended use is not permitted by statutory regulation or exceeds the permitted use, you will need to obtain permission directly from the copyright holder. To view a copy of this licence, visit http://creativecommons.org/licenses/by/4.0/.

\section{References}

1. Cellura E, Spataro R, Taiello AC, La Bella V (2012) Factors affecting the diagnostic delay in amyotrophic lateral sclerosis. Clin Neurol Neurosurg 114(6):550-554. https://doi.org/10.1016/j.cline uro.2011.11.026

2. Chio A, Logroscino G, Hardiman O, Swingler R, Mitchell D, Beghi E, Traynor BG (2009) Prognostic factors in ALS: a critical review. Amyotroph Lateral Scler 10(5-6):310-323. https://doi. org/10.3109/17482960802566824

3. Czaplinski A, Yen AA, Appel SH (2006) Amyotrophic lateral sclerosis: early predictors of prolonged survival. J Neurol 253(11):1428-1436. https://doi.org/10.1007/s00415-006-0226-8 
4. Donaghy C, Dick A, Hardiman O, Patterson V (2008) Timeliness of diagnosis in motor neurone disease: a population-based study. Ulst Med J 77(1):18-21

5. Schuster C, Elamin M, Hardiman O, Bede P (2015) Presymptomatic and longitudinal neuroimaging in neurodegeneration-from snapshots to motion picture: a systematic review. J Neurol Neurosurg Psychiatry 86(10):1089-1096. https://doi.org/10.1136/ jnnp-2014-309888

6. Chipika RH, Finegan E, Li Hi Shing S, Hardiman O, Bede P (2019) Tracking a fast-moving disease: longitudinal markers, monitoring, and clinical trial endpoints in ALS. Front Neurol 10:229. https://doi.org/10.3389/fneur.2019.00229

7. Bede P, Hardiman O (2018) Longitudinal structural changes in ALS: a three time-point imaging study of white and gray matter degeneration. Amyotroph Lateral Scler Frontotemporal Degener 19(3-4):232-241. https://doi.org/10.1080/21678421.2017.14077 95

8. Bertrand A, Wen J, Rinaldi D, Houot M, Sayah S, Camuzat A, Fournier C, Fontanella S, Routier A, Couratier P, Pasquier F, Habert MO, Hannequin D, Martinaud O, Caroppo P, Levy R, Dubois B, Brice A, Durrleman S, Colliot O, Le Ber I (2018) Early cognitive, structural, and microstructural changes in presymptomatic C9orf72 carriers younger than 40 years. JAMA Neurol 75(2):236-245. https://doi.org/10.1001/jamaneurol.2017.4266

9. Wen J, Zhang H, Alexander DC, Durrleman S, Routier A, Rinaldi D, Houot M, Couratier P, Hannequin D, Pasquier F, Zhang J, Colliot O, Le Ber I, Bertrand A (2019) Neurite density is reduced in the presymptomatic phase of C9orf72 disease. J Neurol Neurosurg Psychiatry 90(4):387-394. https://doi.org/10.1136/ jnnp-2018-318994

10. Chipika RH, Siah WF, McKenna MC, Li Hi Shing S, Hardiman O, Bede P (2020) The presymptomatic phase of amyotrophic lateral sclerosis: are we merely scratching the surface? J Neurol. https:// doi.org/10.1007/s00415-020-10289-5

11. Bede P, Siah WF, McKenna MC, Li Hi Shing S (2020) Consideration of C9orf72-associated ALS-FTD as a neurodevelopmental disorder: insights from neuroimaging. J Neurol Neurosurg Psychiatry. https://doi.org/10.1136/jnnp-2020-324416

12. Agosta F, Spinelli EG, Filippi M (2018) Neuroimaging in amyotrophic lateral sclerosis: current and emerging uses. Expert Rev Neurother 18(5):395-406. https://doi.org/10.1080/14737175. 2018.1463160

13. Agosta F, Ferraro PM, Riva N, Spinelli EG, Chio A, Canu E, Valsasina P, Lunetta C, Iannaccone S, Copetti M, Prudente E, Comi G, Falini A, Filippi M (2016) Structural brain correlates of cognitive and behavioral impairment in MND. Hum Brain Mapp 37(4):1614-1626. https://doi.org/10.1002/hbm.23124

14. Muller HP, Turner MR, Grosskreutz J, Abrahams S, Bede P, Govind V, Prudlo J, Ludolph AC, Filippi M, Kassubek J (2016) A large-scale multicentre cerebral diffusion tensor imaging study in amyotrophic lateral sclerosis. J Neurol Neurosurg Psychiatry 87(6):570-579. https://doi.org/10.1136/jnnp-2015-311952

15. Agosta F, Spinelli EG, Marjanovic IV, Stevic Z, Pagani E, Valsasina P, Salak-Djokic B, Jankovic M, Lavrnic D, Kostic VS, Filippi M (2018) Unraveling ALS due to SOD1 mutation through the combination of brain and cervical cord MRI. Neurology. https://doi.org/10.1212/wnl.0000000000005002

16. Ferraro PM, Agosta F, Riva N, Copetti M, Spinelli EG, Falzone Y, Sorarù G, Comi G, Chiò A, Filippi M (2017) Multimodal structural MRI in the diagnosis of motor neuron diseases. NeuroImage Clin 16:240-247. https://doi.org/10.1016/j.nicl.2017.08.002

17. Grollemund V, Pradat PF, Querin G, Delbot F, Le Chat G, PradatPeyre JF, Bede P (2019) Machine learning in amyotrophic lateral sclerosis: achievements, pitfalls, and future directions. Front Neurosci 13:135. https://doi.org/10.3389/fnins.2019.00135
18. Grollemund V, Le Chat G, Secchi-Buhour MS, Delbot F, PradatPeyre JF, Bede P, Pradat PF (2020) Manifold learning for amyotrophic lateral sclerosis functional loss assessment : Development and validation of a prognosis model. J Neurol. https://doi.org/10. 1007/s00415-020-10181-2

19. Bede P, Querin G, Pradat PF (2018) The changing landscape of motor neuron disease imaging: the transition from descriptive studies to precision clinical tools. Curr Opin Neurol 31(4):431438. https://doi.org/10.1097/wco.0000000000000569

20. Schuster C, Hardiman O, Bede P (2016) Development of an automated MRI-based diagnostic protocol for amyotrophic lateral sclerosis using disease-specific pathognomonic features: a quantitative disease-state classification study. PLoS ONE 11(12):e0167331. https://doi.org/10.1371/journal.pone.0167331

21. Welsh RC, Jelsone-Swain LM, Foerster BR (2013) The utility of independent component analysis and machine learning in the identification of the amyotrophic lateral sclerosis diseased brain. Front Hum Neurosci 7:251. https://doi.org/10.3389/ fnhum.2013.00251

22. Bede P, Iyer PM, Finegan E, Omer T, Hardiman O (2017) Virtual brain biopsies in amyotrophic lateral sclerosis: diagnostic classification based on in vivo pathological patterns. NeuroImage Clin 15:653-658. https://doi.org/10.1016/j.nicl.2017.06.010

23. Tahedl M, Chipika RH, Lope J, Li Hi Shing S, Hardiman O, Bede P (2021) Cortical progression patterns in individual ALS patients across multiple timepoints: a mosaic-based approach for clinical use. J Neurol 268(5):1913-1926. https://doi.org/10. 1007/s00415-020-10368-7

24. Tahedl M, Murad A, Lope J, Hardiman O, Bede P (2021) Evaluation and categorisation of individual patients based on white matter profiles: single-patient diffusion data interpretation in neurodegeneration. J Neurol Sci 428:117584. https://doi.org/ 10.1016/j.jns.2021.117584

25. Fischl B (2012) FreeSurfer. Neuroimage 62(2):774-781. https:// doi.org/10.1016/j.neuroimage.2012.01.021

26. Fischl B, Dale AM (2000) Measuring the thickness of the human cerebral cortex from magnetic resonance images. Proc Natl Acad Sci 97(20):11050-11055. https://doi.org/10.1073/ pnas. 200033797

27. Iglesias JE, Van Leemput K, Bhatt $P$, Casillas $C$, Dutt $S$, Schuff $N$, Truran-Sacrey D, Boxer A, Fischl B (2015) Bayesian segmentation of brainstem structures in MRI. Neuroimage 113:184-195. https://doi.org/10.1016/j.neuroimage.2015.02.065

28. Mori S, Oishi K, Jiang H, Jiang L, Li X, Akhter K, Hua K, Faria AV, Mahmood A, Woods R, Toga AW, Pike GB, Neto PR, Evans A, Zhang J, Huang H, Miller MI, van Zijl P, Mazziotta J (2008) Stereotaxic white matter atlas based on diffusion tensor imaging in an ICBM template. Neuroimage 40(2):570-582. https://doi.org/ 10.1016/j.neuroimage.2007.12.035

29. Mori S, Wakana S, Van Zijl P, Nagae-Poetscher L (2005) MRI atlas of human white matter. Elsevier, The Netherlands

30. Wakana S, Caprihan A, Panzenboeck MM, Fallon JH, Perry M, Gollub RL, Hua K, Zhang J, Jiang H, Dubey P, Blitz A, van Zijl P, Mori S (2007) Reproducibility of quantitative tractography methods applied to cerebral white matter. Neuroimage 36(3):630-644. https://doi.org/10.1016/j.neuroimage.2007.02.049

31. Hua K, Zhang J, Wakana S, Jiang H, Li X, Reich DS, Calabresi PA, Pekar JJ, van Zijl PC, Mori S (2008) Tract probability maps in stereotaxic spaces: analyses of white matter anatomy and tractspecific quantification. Neuroimage 39(1):336-347. https://doi. org/10.1016/j.neuroimage.2007.07.053

32. Collins DL, Holmes CJ, Peters TM, Evans AC (1995) Automatic 3-D model-based neuroanatomical segmentation. Hum Brain Mapp 3(3):190-208. https://doi.org/10.1002/hbm.460030304

33. Mazziotta J, Toga A, Evans A, Fox P, Lancaster J, Zilles K, Woods R, Paus T, Simpson G, Pike B, Holmes C, Collins L, Thompson P, 
MacDonald D, Iacoboni M, Schormann T, Amunts K, PalomeroGallagher N, Geyer S, Parsons L, Narr K, Kabani N, Le Goualher G, Boomsma D, Cannon T, Kawashima R, Mazoyer B (2001) A probabilistic atlas and reference system for the human brain: International Consortium for Brain Mapping (ICBM). Philos Trans R Soc Lond B Biol Sci 356(1412):1293-1322. https://doi.org/10.1098/rstb.2001.0915

34. Brown CA, Johnson NF, Anderson-Mooney AJ, Jicha GA, Shaw LM, Trojanowski JQ, Van Eldik LJ, Schmitt FA, Smith CD, Gold BT (2017) Development, validation and application of a new fornix template for studies of aging and preclinical Alzheimer's disease. NeuroImage Clin 13:106-115. https://doi.org/10.1016/j. nicl.2016.11.024

35. Grollemund V, Chat GL, Secchi-Buhour MS, Delbot F, PradatPeyre JF, Bede P, Pradat PF (2020) Development and validation of a 1-year survival prognosis estimation model for Amyotrophic Lateral Sclerosis using manifold learning algorithm UMAP. Sci Rep 10(1):13378. https://doi.org/10.1038/s41598-020-70125-8

36. Schuster C, Hardiman O, Bede P (2017) Survival prediction in Amyotrophic lateral sclerosis based on MRI measures and clinical characteristics. BMC Neurol 17(1):73. https://doi.org/10.1186/ s12883-017-0854-x

37. Nie D, Trullo R, Lian J, Wang L, Petitjean C, Ruan S, Wang Q, Shen D (2018) Medical image synthesis with deep convolutional adversarial networks. IEEE Trans Biomed Eng 65(12):2720-2730. https://doi.org/10.1109/tbme.2018.2814538

38. Amato F, López A, Peña-Méndez E, VbHhara P, Hampl A, Havel J (2013) Artificial neural networks in medical diagnosis. J Appl Biomed 11:47-58

39. Lisboa PJ, Taktak AF (2006) The use of artificial neural networks in decision support in cancer: a systematic review. Neural Netw 19(4):408-415. https://doi.org/10.1016/j.neunet.2005.10.007

40. Suzuki K (2017) Overview of deep learning in medical imaging. Radiol Phys Technol 10(3):257-273. https://doi.org/10.1007/ s12194-017-0406-5

41. Menke RAL, Proudfoot M, Talbot K, Turner MR (2018) The two-year progression of structural and functional cerebral MRI in amyotrophic lateral sclerosis. NeuroImage Clin 17:953-961. https://doi.org/10.1016/j.nicl.2017.12.025

42. Bede P, Chipika RH, Finegan E, Li Hi Shing S, Doherty MA, Hengeveld JC, Vajda A, Hutchinson S, Donaghy C, McLaughlin RL, Hardiman O (2019) Brainstem pathology in amyotrophic lateral sclerosis and primary lateral sclerosis: a longitudinal neuroimaging study. NeuroImage Clin 24:102054. https://doi.org/10.1016/j. nicl.2019.102054

43. Schuster C, Elamin M, Hardiman O, Bede P (2016) The segmental diffusivity profile of amyotrophic lateral sclerosis associated white matter degeneration. Eur J Neurol 23(8):1361-1371. https://doi. org/10.1111/ene.13038

44. Abidi M, de Marco G, Couillandre A, Feron M, Mseddi E, Termoz N, Querin G, Pradat PF, Bede P (2020) Adaptive functional reorganization in amyotrophic lateral sclerosis: coexisting degenerative and compensatory changes. Eur J Neurol 27(1):121-128. https://doi.org/10.1111/ene.14042

45. Abidi M, de Marco G, Grami F, Termoz N, Couillandre A, Querin G, Bede P, Pradat PF (2021) Neural correlates of motor imagery of gait in amyotrophic lateral sclerosis. J Magn Reson Imaging 53(1):223-233. https://doi.org/10.1002/jmri.27335

46. Feron M, Couillandre A, Mseddi E, Termoz N, Abidi M, Bardinet E, Delgadillo D, Lenglet T, Querin G, Welter ML, Le Forestier N, Salachas F, Bruneteau G, Del Mar AM, Debs R, Lacomblez L, Meininger V, Pelegrini-Issac M, Bede P, Pradat PF, de Marco G (2018) Extrapyramidal deficits in ALS: a combined biomechanical and neuroimaging study. J Neurol 265(9):2125-2136. https://doi. org/10.1007/s00415-018-8964-y

47. McKenna MC, Chipika RH, Li Hi Shing S, Christidi F, Lope J, Doherty MA, Hengeveld JC, Vajda A, McLaughlin RL,
Hardiman O, Hutchinson S, Bede P (2021) Infratentorial pathology in frontotemporal dementia: cerebellar grey and white matter alterations in FTD phenotypes. J Neurol. https://doi.org/10.1007/ s00415-021-10575-w

48. Bede P, Chipika RH, Christidi F, Hengeveld JC, Karavasilis E, Argyropoulos GD, Lope J, Li Hi Shing S, Velonakis G, Dupuis L, Doherty MA, Vajda A, McLaughlin RL, Hardiman O (2021) Genotype-associated cerebellar profiles in ALS: focal cerebellar pathology and cerebro-cerebellar connectivity alterations. J Neurol Neurosurg Psychiatry. https://doi.org/10.1136/ jnnp-2021-326854

49. Bharti K, Khan M, Beaulieu C, Graham SJ, Briemberg H, Frayne R, Genge A, Korngut L, Zinman L, Kalra S (2020) Involvement of the dentate nucleus in the pathophysiology of amyotrophic lateral sclerosis: a multi-center and multi-modal neuroimaging study. NeuroImage Clin 28:102385. https://doi. org/10.1016/j.nicl.2020.102385

50. Tu S, Menke RAL, Talbot K, Kiernan MC, Turner MR (2019) Cerebellar tract alterations in PLS and ALS. Amyotroph Lateral Scler Frontotemporal Degener 20(3-4):281-284. https://doi.org/ 10.1080/21678421.2018.1562554

51. Christidi F, Karavasilis E, Rentzos M, Kelekis N, Evdokimidis I, Bede P (2018) Clinical and radiological markers of extra-motor deficits in amyotrophic lateral sclerosis. Front Neurol 9:1005. https://doi.org/10.3389/fneur.2018.01005

52. Agosta F, Galantucci S, Magnani G, Marcone A, Martinelli D, Antonietta Volonte M, Riva N, Iannaccone S, Ferraro PM, Caso F, Chio A, Comi G, Falini A, Filippi M (2015) MRI signatures of the frontotemporal lobar degeneration continuum. Hum Brain Mapp 36(7):2602-2614. https://doi.org/10.1002/hbm.22794

53. Bede P, Omer T, Finegan E, Chipika RH, Iyer PM, Doherty MA, Vajda A, Pender N, McLaughlin RL, Hutchinson S, Hardiman O (2018) Connectivity-based characterisation of subcortical grey matter pathology in frontotemporal dementia and ALS: a multimodal neuroimaging study. Brain Imaging Behav 12(6):16961707. https://doi.org/10.1007/s11682-018-9837-9

54. Bede P, Iyer PM, Schuster C, Elamin M, McLaughlin RL, Kenna K, Hardiman O (2016) The selective anatomical vulnerability of ALS: "disease-defining" and "disease-defying" brain regions. Amyotroph Lateral Scler Frontotemporal Degener 17(7-8):561570. https://doi.org/10.3109/21678421.2016.1173702

55. Blasco H, Patin F, Descat A, Garcon G, Corcia P, Gele P, Lenglet T, Bede P, Meininger V, Devos D, Gossens JF, Pradat PF (2018) A pharmaco-metabolomics approach in a clinical trial of ALS: identification of predictive markers of progression. PLoS ONE 13(6):e0198116. https://doi.org/10.1371/journal.pone.0198116

56. Devos D, Moreau C, Kyheng M, Garcon G, Rolland AS, Blasco H, Gele P, Timothee Lenglet T, Veyrat-Durebex C, Corcia P, Dutheil M, Bede P, Jeromin A, Oeckl P, Otto M, Meninger V, Danel-Brunaud V, Devedjian JC, Duce JA, Pradat PF (2019) A ferroptosis-based panel of prognostic biomarkers for Amyotrophic Lateral Sclerosis. Sci Rep 9(1):2918. https://doi.org/10.1038/ s41598-019-39739-5

57. Dukic S, McMackin R, Buxo T, Fasano A, Chipika R, Pinto-Grau M, Costello E, Schuster C, Hammond M, Heverin M, Coffey A, Broderick M, Iyer PM, Mohr K, Gavin B, Pender N, Bede P, Muthuraman M, Lalor EC, Hardiman O, Nasseroleslami B (2019) Patterned functional network disruption in amyotrophic lateral sclerosis. Hum Brain Mapp 40(16):4827-4842. https://doi.org/ $10.1002 / \mathrm{hbm} .24740$

58. Nasseroleslami B, Dukic S, Broderick M, Mohr K, Schuster C, Gavin B, McLaughlin R, Heverin M, Vajda A, Iyer PM, Pender N, Bede P, Lalor EC, Hardiman O (2019) Characteristic increases in EEG connectivity correlate with changes of structural MRI in amyotrophic lateral sclerosis. Cereb Cortex 29(1):27-41. https:// doi.org/10.1093/cercor/bhx301 
59. Iyer PM, Mohr K, Broderick M, Gavin B, Burke T, Bede P, PintoGrau M, Pender NP, McLaughlin R, Vajda A, Heverin M, Lalor EC, Hardiman O, Nasseroleslami B (2017) Mismatch negativity as an indicator of cognitive sub-domain dysfunction in amyotrophic lateral sclerosis. Front Neurol 8:395. https://doi.org/10. 3389/fneur.2017.00395

60. Proudfoot M, Bede P, Turner MR (2018) Imaging cerebral activity in amyotrophic lateral sclerosis. Front Neurol 9:1148. https://doi. org/10.3389/fneur.2018.01148

61. Verstraete E, Turner MR, Grosskreutz J, Filippi M, Benatar M (2015) Mind the gap: the mismatch between clinical and imaging metrics in ALS. Amyotroph Lateral Scler Frontotemporal Degener 16(7-8):524-529. https://doi.org/10.3109/21678421.2015.10519 89

62. Burke T, Pinto-Grau M, Lonergan K, Elamin M, Bede P, Costello E, Hardiman O, Pender N (2016) Measurement of social cognition in amyotrophic lateral sclerosis: a population based study. PLoS ONE 11(8):e0160850. https://doi.org/10.1371/journal.pone.0160850

63. Burke T, Elamin M, Bede P, Pinto-Grau M, Lonergan K, Hardiman O, Pender N (2016) Discordant performance on the "Reading the Mind in the Eyes" Test, based on disease onset in amyotrophic lateral sclerosis. Amyotroph Lateral Scler Frontotemporal Degener. https://doi.org/10.1080/21678421.2016.1177088

64. Finegan E, Chipika RH, Li Hi Shing S, Hardiman O, Bede P (2019) Crying and laughing in motor neuron disease: pathobiology, screening, intervention. Front Neurol 10:260. https://doi.org/ 10.3389/fneur.2019.00260

65. Querin G, El Mendili MM, Bede P, Delphine S, Lenglet T, Marchand-Pauvert V, Pradat PF (2018) Multimodal spinal cord MRI offers accurate diagnostic classification in ALS. J Neurol Neurosurg Psychiatry 89(11):1220-1221. https://doi.org/10.1136/ jnnp-2017-317214

66. El Mendili MM, Querin G, Bede P, Pradat PF (2019) Spinal cord imaging in amyotrophic lateral sclerosis: historical concepts-novel techniques. Front Neurol 10:350. https://doi.org/10.3389/fneur. 2019.00350

67. Querin G, El Mendili MM, Lenglet T, Behin A, Stojkovic T, Salachas F, Devos D, Le Forestier N, Del Mar AM, Debs R, Lacomblez L, Meninger V, Bruneteau G, Cohen-Adad J, Lehericy S, Laforet P, Blancho S, Benali H, Catala M, Li M, MarchandPauvert V, Hogrel JY, Bede P, Pradat PF (2019) The spinal and cerebral profile of adult spinal-muscular atrophy: a multimodal imaging study. NeuroImage Clin 21:101618. https://doi.org/10. 1016/j.nicl.2018.101618

68. Valsasina P, Agosta F, Benedetti B, Caputo D, Perini M, Salvi F, Prelle A, Filippi M (2007) Diffusion anisotropy of the cervical cord is strictly associated with disability in amyotrophic lateral sclerosis. J Neurol Neurosurg Psychiatry 78(5):480-484. https:// doi.org/10.1136/jnnp.2006.100032

69. Finegan E, Chipika RH, Shing SLH, Hardiman O, Bede P (2019) Primary lateral sclerosis: a distinct entity or part of the ALS spectrum? Amyotroph Lateral Scler Frontotemporal Degener 20(34):133-145. https://doi.org/10.1080/21678421.2018.1550518

70. Finegan E, Li Hi Shing S, Siah WF, Chipika RH, Chang KM, McKenna MC, Doherty MA, Hengeveld JC, Vajda A, Donaghy C, Hutchinson S, McLaughlin RL, Hardiman O, Bede P (2020) Evolving diagnostic criteria in primary lateral sclerosis: the clinical and radiological basis of "probable PLS." J Neurol Sci 417:117052. https://doi.org/10.1016/j.jns.2020.117052

71. Finegan E, Chipika RH, Li Hi Shing S, Doherty MA, Hengeveld JC, Vajda A, Donaghy C, McLaughlin RL, Pender N, Hardiman O, Bede P (2019) The clinical and radiological profile of primary lateral sclerosis: a population-based study. J Neurol 266(11):2718-2733. https://doi.org/10.1007/s00415-019-09473-Z

72. Christidi F, Karavasilis E, Rentzos M, Velonakis G, Zouvelou V, Xirou S, Argyropoulos G, Papatriantafyllou I, Pantolewn V,
Ferentinos P, Kelekis N, Seimenis I, Evdokimidis I, Bede P (2019) Hippocampal pathology in amyotrophic lateral sclerosis: selective vulnerability of subfields and their associated projections. Neurobiol Aging 84:178-188. https://doi.org/10.1016/j.neurobiolaging. 2019.07.019

73. Finegan E, Li Hi Shing S, Chipika RH, Doherty MA, Hengeveld JC, Vajda A, Donaghy C, Pender N, McLaughlin RL, Hardiman O, Bede P (2019) Widespread subcortical grey matter degeneration in primary lateral sclerosis: a multimodal imaging study with genetic profiling. NeuroImage Clin 24:102089. https://doi.org/10. 1016/j.nicl.2019.102089

74. Chipika RH, Finegan E, Li Hi Shing S, McKenna MC, Christidi F, Chang KM, Doherty MA, Hengeveld JC, Vajda A, Pender N, Hutchinson S, Donaghy C, McLaughlin RL, Hardiman O, Bede P (2020) "Switchboard" malfunction in motor neuron diseases: selective pathology of thalamic nuclei in amyotrophic lateral sclerosis and primary lateral sclerosis. NeuroImage Clin 27:102300. https://doi.org/10.1016/j.nicl.2020.102300

75. Chipika RH, Christidi F, Finegan E, Li Hi Shing S, McKenna MC, Chang KM, Karavasilis E, Doherty MA, Hengeveld JC, Vajda A, Pender N, Hutchinson S, Donaghy C, McLaughlin RL, Hardiman O, Bede P (2020) Amygdala pathology in amyotrophic lateral sclerosis and primary lateral sclerosis. J Neurol Sci. https://doi. org/10.1016/j.jns.2020.117039

76. Lebouteux MV, Franques J, Guillevin R, Delmont E, Lenglet T, Bede P, Desnuelle C, Pouget J, Pascal-Mousselard H, Pradat PF (2014) Revisiting the spectrum of lower motor neuron diseases with snake eyes appearance on magnetic resonance imaging. Eur J Neurol 21(9):1233-1241. https://doi.org/10.1111/ene.12465

77. Querin G, Lenglet T, Debs R, Stojkovic T, Behin A, Salachas F, Le Forestier N, Amador MDM, Bruneteau G, Laforêt P, Blancho S, Marchand-Pauvert V, Bede P, Hogrel JY, Pradat PF (2021) Development of new outcome measures for adult SMA type III and IV: a multimodal longitudinal study. J Neurol. https://doi.org/ 10.1007/s00415-020-10332-5

78. Querin G, Bede P, Marchand-Pauvert V, Pradat PF (2018) Biomarkers of spinal and bulbar muscle atrophy (SBMA): a comprehensive review. Front Neurol 9:844. https://doi.org/10.3389/fneur. 2018.00844

79. Pradat PF, Bernard E, Corcia P, Couratier P, Jublanc C, Querin G, Morelot Panzini C, Salachas F, Vial C, Wahbi K, Bede P, Desnuelle C (2020) The French national protocol for Kennedy's disease (SBMA): consensus diagnostic and management recommendations. Orphanet J Rare Dis 15(1):90. https://doi.org/10. 1186/s13023-020-01366-z

80. Li Hi Shing S, Chipika RH, Finegan E, Murray D, Hardiman O, Bede P (2019) Post-polio syndrome: more than just a lower motor neuron disease. Front Neurol 10:773. https://doi.org/10. 3389/fneur.2019.00773

81. Spinelli EG, Agosta F, Ferraro PM, Querin G, Riva N, Bertolin C, Martinelli I, Lunetta C, Fontana A, Sorarù G, Filippi M (2019) Brain MRI shows white matter sparing in Kennedy's disease and slow-progressing lower motor neuron disease. Hum Brain Mapp 40(10):3102-3112. https://doi.org/10.1002/hbm.24583

82. Spinelli EG, Agosta F, Ferraro PM, Riva N, Lunetta C, Falzone YM, Comi G, Falini A, Filippi M (2016) Brain MR imaging in patients with lower motor neuron-predominant disease. Radiology 280(2):545-556. https://doi.org/10.1148/radiol.2016151846

83. Querin G, Bede P, El Mendili MM, Li M, Pelegrini-Issac M, Rinaldi D, Catala M, Saracino D, Salachas F, Camuzat A, Marchand-Pauvert V, Cohen-Adad J, Colliot O, Le Ber I, Pradat PF (2019) Presymptomatic spinal cord pathology in c9orf72 mutation carriers: a longitudinal neuroimaging study. Ann Neurol 86(2):158-167. https://doi.org/10.1002/ana.25520 